\title{
Responsiveness of the Short Warwick Edinburgh Mental Well-Being Scale (SWEMWBS): evaluation a clinical sample
}

\author{
Neha Shah ${ }^{1 *}$, Mizaya Cader ${ }^{2}$, William P. Andrews ${ }^{3}$, Dilini Wijesekera ${ }^{2}$ and Sarah L. Stewart-Brown ${ }^{4}$
}

\begin{abstract}
Background: SWEMWBS is a popular measure of mental wellbeing, shown to be valid in clinical populations. Responsiveness to change has not yet been formally assessed.

Methods: Analysis of data from a clinical sample of 172 clients undergoing up to 4 sessions of cognitive hypnotherapy. Cohen's D effect size (ES), Standardised response mean (SRM), probability of change statistic $(P \wedge)$ were used to evaluate whether SWEMWBS detected statistically important changes at the group level. Cohen's D effect size (ES) and Standard error of measurement (SEM) and were used to evaluate whether SWEMWBS detected statistically important changes at the individual level.

Results: Mean (SD) SWEMWBS scores increased from baseline to therapy 4 from 19.28 (3.921) to 23.32 (4.873). At group level, using Cohen's D effect size, improvement ranges from ES =0.20-1.41 and using SRM, ranged from 0.30-0. 88 , increasing with number of therapy sessions. $(P \wedge)$ ranged from $0.65-0.8$. At individual level, use of Cohens $D$ ES $>0.5$ indicated statistically important improvement in $29.9-86.1 \%$ cf. 20.1-80.6\% using a standard of 2.77 SEM (2.87 points). The lower threshold of 1 SEM (1.03 points) indicated statistically important improvement in 43.0-81.0\%.

Conclusion: SWEMWBS is responsive to change at individual and group level. At individual level a change of between 1 and 3 points meets thresholds for statisticially important change, depending on standard used. Anchor based studies are necessary to confirm that such change represents minimally important change from the perspective of study participants.
\end{abstract}

\section{Background}

Mental wellbeing, the positive aspect of mental health, is a core concept for public mental health and mental health promotion [1]. The Warwick-Edinburgh Mental Well-Being Scale (WEMWBS) was developed in 2007 to support public mental health by enabling the monitoring of mental wellbeing, investigation of determinants and evaluation of interventions [2]. The conceptual framework behind WEMWBS reflects growing consensus that mental wellbeing consists of two key dimensions: feeling good or hedonia, and functioning well or eudaimonia $[3,4]$. A seven item version, the Short Warwick-Edinburgh Mental Well-Being Scale (SWEMWBS) was resolved in 2009 using Rasch modelling. This offers

\footnotetext{
* Correspondence: N.Shah.5@warwick.ac.uk

${ }^{1}$ Public Health Registrar, Warwick Medical School University of Warwick, Coventry, UK

Full list of author information is available at the end of the article
}

superior interval scaling to WEMWBS [5]. Robust measurement properties combined with brevity make SWEMWBS popular for monitoring mental wellbeing in populations.

Mental wellbeing is now also beginning to be recognised as an outcome of importance in mental health services [6] where valid outcome measures, usually based on patient self-report, are required by commissioners to monitor the effectiveness of service provision [7]. These measures may also be valuable to clinicians for monitoring treatment progress and aiding clinical decision-making [8]. Research has suggested that WEMWBS and SWEMWBS are well liked by service users and carers, who value the positive wording, self-administered nature and appropriate length [6]. Whilst WEMWBS responsiveness to change has been demonstrated [9], responsiveness of SWEMWBS to change in clinical and community settings has not been formally evaluated. 
Responsiveness covers an instrument's ability to accurately detect meaningful change when change occurs. Minimal important change can be defined as minimum change which is of significance to the patient, member of the public or the health professional, that exceeds variation attributable to chance $[10,11-13]$. There are two broad methods for measuring responsiveness: distribution based, where the observed change is compared to the statistical properties of the sample, which measures variation attributable to chance; and instrument or anchor based, where the observed change is related to an external criterion of change and in clinical populations measures clinical significance [14]. Meaningful change can be different at group and individual level.

The aim of this study was to evaluate the responsiveness of SWEMWBS using distributional methods in a clinical sample of cognitive hypnotherapy service users at group and individual level, and thus provide further evidence regarding its' suitability as an outcome measure in clinical practice.

\section{Methods}

Data for this study were collected by cognitive hypnotherapists at Quest Cognitive Hypnotherapy (QCH) during routine clinical practice using the Pragmatic Research Network's electronic software (Pragmatic Tracker) [15]. The latter is a collaboration of professionals promoting service-based evaluation and feedback-informed treatment which developed Pragmatic Tracker to allow session by session administration of outcome measures, with feedback to clients, therapists and service evaluators.

Therapists were informed about the proposed study through the Quest online forum by the project coordinator and were invited to participate in the research project. All therapists worked in private practice with fee-paying clients in a range of locations throughout the UK. The research project was overseen by the pragmatic research network providing an initial training day and combination of face-to-face, telephone and e-mail support.

\section{Participants}

Participants were adult clients seeking cognitive hypnotherapy $(\mathrm{CHT})$ as treatment for mental health problems, mainly anxiety and depression, at the $\mathrm{QCH}$ practices of the participating therapists between October 2014 and April 2016. 167 participants were recruited at initial session, 36 of whom provided data for 4 sessions.

\section{Intervention}

$\mathrm{CHT}$ is a type of therapy which uses induction of the patient into a trance like state to access unconscious problematic thoughts, feelings and memory patterns. At the initial assessment, the therapist identifies the client's use of language and the unconscious phenomena they experience while acting within their problem pattern. Subsequent sessions build on these findings focusing on interrupting faulty pattern matching by changing the context, structure, process or consequence (the four quadrants) of the problem pattern. Each technique or intervention acts within a specific quadrant, so treatment is highly individualised, based on the content of the client's unique problem pattern [15]. There is no set amount of treatment sessions; length and frequency of treatment is negotiated between therapist and client based upon progress and ongoing need.

\section{Outcome measure}

SWEMWBS was selected as one of several outcome measures for inclusion in the pragmatic tracker software because therapists found it helpful to monitor treatment from a positive perspective. Because intervals between sessions are often one week long, SWEMWBS was presented with a one rather than two week time frame for response options All participants were informed about QCH research objectives and written consent for outcome monitoring was obtained at the first session. Individual client data were gathered before assessment and at each subsequent session by self-administration using web based 'pragmatic tracker' software, either remotely via email link or on arrival at the clinic.

\section{Statistical analysis}

Analyses were performed using the SPSS (v23.0, IBM) and MedCalc (version 17.9, MedCalc Software) packages. Normality of distribution for SWEMWBS across participants was assessed by visual inspection of the histogram and using the Shapiro-Wilk test for normality. Descriptive statistics including the mean and standard deviation (SD) of SWEMWBS score at each session were calculated.

There is no clear consensus regarding which statistical standards should be used to assess responsiveness. We used four distributional methods [11, 13, 14, 16]: Cohen's D effect size and Standardized Response Mean (SRM) for group level analysis $[9,11,17]$ and Cohen's D effect size and Standard Error of the Mean (SEM) for individual level analysis $[10,18]$.

\section{Group level analysis}

Distribution of scores was investigated using a paired $t$ test for group level analysis between assessment and each time point up to 4 therapy sessions. Cohen's D was calculated by dividing the mean difference of paired measurements between assessment and each time point by the pooled Standard Deviation (SD) of assessment and the respective time point. The standard cut off values for Cohen's D; 'trivial' $(E S<0.20)$, 'small' $(E S \geq 0.20<0.50)$, 'moderate' 
( $E S \geq 0.50<0.80$ ), or large ( $E S \geq 0.80$ ) were used to describe statistically meaningful change at group level [17].

SRM was calculated by dividing the mean difference of paired measurements between assessment and each time point by the standard deviation of the differences between the paired measurements [11]. SRM was interpreted by calculating the probability of change statistic P, which relates to the cumulative normal distribution function of the derived SRM. The P statistic denotes the probability that the instrument detects a change, intuitively representing the proportion of subjects whose scores have changed, and ranges from 0.5 (no ability to detect change) to 1 (perfect ability to detect change) [19]. The $95 \%$ CI of the P statistic was estimated using the substitution method, which uses the cumulative standard normal distribution function of the SRM to calculate the respective lower and upper limit [20]. 95\% confidence intervals for the Cohen's D and SRM were calculated using bootstrapping with 1000 different combinations from the existing data to derive lower and upper limit.

\section{Individual level analysis}

Cohen's D was calculated for every individual by dividing the difference between assessment score and score at each therapy session up to 4 sessions by the pooled SD of assessment and respective session score. A threshold of ES $>0.5$ was chosen as a cut off for statistically meaningful change at individual level, as recommended by Norman et al. after a review of various distributional and anchor based methods for establishing minimal important difference [18]. The proportion of patients with improvement after each therapy session was calculated.

SEM of the instrument was calculated as; $\mathrm{SEM}=\mathrm{SD}$ (baseline) $* \sqrt{1}$-rxx (internal consistency reliability of the instrument). Cronbach's alpha was used to calculate the reliability statistic. Different thresholds ranging from 1 SEM to 2.77 SEM have been proposed to consider individual level change as statistically meaningful [21]. As the SEM of a measure is said to be independent of the sample [21], a single value change value can be applied to denote improvement across different samples. A threshold of 2.77 was chosen which takes into account measurement error, the combined variability across baseline and post intervention samples, and chance at the $95 \%$ confidence interval [22]. The proportion of individuals with change score more than 2.77 SEM was calculated for each therapy session to determine the proportion with statistically meaningful improvement. Given discrepancy between the two approaches, kappa statistics were used to analyse method agreement [23]. For comparison levels of statistically meaningful change were also calculated using thresholds of 1 SEM and 2 SEM.

\section{Results}

The mean age of the participants was 40.6 years $(N=172$; $\mathrm{SD}=12.71)$. The majority, $74.4 \%(n=128)$ were female, white British $(73.8 \%, N=127)$ and employed $(75.6 \%$, $N=130$ ). 167 participants completed SWEMWBS at assessment; of these 134 of completed data before the first therapy session, 95 before the second, 66 the third and 36 the fourth. There was a mean duration of 12 days between assessment and session 1, 13 days between sessions 1 and 2, 21 days between sessions 2 and 3, and 22 days between sessions 3 and 4. The mean value of SWEMWBS at the assessment session was 19.28 ( $\mathrm{SD}=3.921)$. No significant difference in baseline SWEMWBS was found between groups of clients that attended one, two three and four sessions respectively. Scores increased linearly at each therapy session to reach a mean value of 23.32 $(\mathrm{SD}=4.873)$ before therapy session 4 (see Table 1). Normality of distribution was confirmed on visual inspection and using Shapiro-Wilk testing.

Group level analysis is shown in Table 2. Using Cohen's D a small change of SWEMWBS was observed from assessment to subsequent therapy session 1 (ES = 0.33 ; $95 \%$ CI $0.20-0.46$ ), a moderate change from assessment to therapy session $2(\mathrm{ES}=0.67 ; 95 \% \mathrm{CI} 0.48-0.86)$ and large changes from assessment to therapy 3 (ES = $0.9295 \% \mathrm{CI} 0.69-1.15)$ and therapy $4(\mathrm{ES}=1.03 ; 95 \% \mathrm{CI}$ $0.60-1.41)$. The SRM ranged from 0.49 (95\% CI 0.30 $0.65)$ to 1.01 (95\% CI $0.63-1.36)$ and the probability of change statistic P from 0.69 (95\% CI; 0.65-0.72) to 0.84 (95\% CI; $0.80-0.88$ ). The lower $95 \%$ CI of probability of detecting a statistically meaningful change was 0.65 from assessment to therapy $1,0.71$ from assessment to therapy 2, 0.77 from assessment to therapy 3 and 0.80 from assessment to therapy 4 .

Table 3 reports the results of individual level analysis, showing the proportion of participants with a large improvement by Cohen's D effect size $(E S>0.5)$ and the proportion of participants with a change $>2.77$ SEM using a Cronbach's alpha score of 0.931 derived from this data, which is comparable to previously calculated reliability statistics. Both approaches indicated that the proportion of patients with a significant improvement increased with the progress of therapy, ranging from $38.1 \%$ (95\% CI; $29.9-46.3$ ) to $72.2 \%$ (95\% CI; $58.3-$ 86.1) when $>0.5$ effect size was used and from $27.6 \%$ (95\% CI; $20.1-35.8$ ) to $66.7 \%$ (95\% CI; 50.0-80.6) when $>2.77$ SEM was used. At each time point the proportion with a large improvement by effect size was 6 to $10 \%$ higher than the proportion with a large improvement using SEM > 2.77 approach. Agreement between methods was examined using Kappa statistics; substantial agreement was shown at therapy 1 (0.795), therapy $2(0.874)$ and therapy 3 (0.784); and moderate agreement at therapy 4 (0.609). 
Table 1 Distribution of mean SWEMWBS score at assessment and after therapy session

\begin{tabular}{lllllll}
\hline & N & Mean & SD & $95 \%$ Cl mean & Min & Max \\
\hline SWEMWBS at Assessment & 167 & 19.28 & 3.921 & $18.66-19.89$ & 7 & 35 \\
SWEMWBS before Therapy 1 & 134 & 20.70 & 4.360 & $19.97-21.38$ & 7 & 7 \\
SWEMWBS before Therapy 2 & 95 & 21.60 & 5.343 & $20.57-22.73$ & 35 \\
SWEMWBS before Therapy 3 & 66 & 22.47 & 4.383 & $21.44-23.48$ & 3.4 \\
SWEMWBS before Therapy 4 & 36 & 23.32 & 4.873 & $21.77-24.85$ & 9.51 \\
\hline
\end{tabular}

Given the discrepancy between the two standards and in line with other literature [21], lower thresholds of 1 and 2 SEM were also examined (see Table 4). These showed improvement in $51.5 \%$ (95\% CI; $43.0-59.8)$ to $72.2 \%$ (95\% CI; 56.0-84.1) at a threshold of 1 SEM, and in $38.1 \%$ (95\% CI; 30.2-46.5) to 77.8\% (95\% CI; 61.9-88.2) at a threshold of 2 SEM. at a threshold of 2 SEM.

\section{Discussion}

\section{Summary of key findings}

English populations norms for SWEMWBS indicate mean (SD) as 23.7 (3.92) for men and 23.2 (3.99) for women [24]. Participants in this study had scores of 19.3 (3.9) at baseline rising to 23.3 (4.9), indicating low mental wellbeing before treatment and, given the predominantly female sample, population average levels after 4 sessions.

Assessment of group level responsiveness using Cohen's $\mathrm{D}$ effect size indicated increasing improvement from assessment at each therapy session $(E S=0.33-1.03$ and using SRM $=0.49-1.01)$. We evaluated the significance of the SRM using the probability of change statistic $\mathrm{P}$ to range between $0.65-0.8$, above 0.5 at every time point, indicating the ability to detect change. This responsiveness can be compared to that of WEMWBS which Maheswaran et al. found to have a probability of change statistic above 0.7 in all studies $[9,25]$. Both methods confirmed that SWEMWBS is able to detect change at group level between each therapy session and that responsiveness increased gradually with each session.
Assessment of individual level responsiveness indicated SWEMWBS ability to detect change at each time point, with improvement of $38.1-72.2 \%$ using Cohen's D as a standard cf. $27.6-66.7 \%$ by 2.77 SEM. Use of the Cohen's D standard overestimated the proportions in comparison to 2.77 SEM at each time point by 6 to $10 \%$. The agreement between two methods was found to be moderate to substantial regardless of this. Further analysis using a lower threshold indicated that Cohens D underestimated ability to detect change compared to a threshold of 1 SEM threshold and approximately equated to ability to detect change at a 2 SEM threshold.

\section{Discussion of methods used}

Cohen's D effect size is dependent on between-subject variability, whilst the SRM is dependent on within-subject variability [26]. A limitation of using effect size as a standard is that it can be influenced by the heterogeneity of the sample, with a larger baseline standard deviation resulting in a smaller effect size. This means that the effect size standard does not account for variation in individual change scores [14]. The SRM approach takes into account the variability in individual change scores. However as a result of this, comparable individual changes have different SRM values depending upon the variability of change in the sample [14]. As SRM and SEM based methods take into account between person differences rather than between group differences, it is likely to be preferable to use SRM and SEM thresholds when looking at before and after change. We found that after

Table 2 Evaluation of responsiveness of SWEMWBS at group level

\begin{tabular}{|c|c|c|c|c|c|c|c|c|c|}
\hline & \multicolumn{6}{|c|}{ Indices of Responsiveness } \\
\hline \multirow{2}{*}{$\begin{array}{l}\text { Mean and SD } \\
\text { Time points }\end{array}$} & \multirow[b]{2}{*}{$\begin{array}{l}\text { Paired mean } \\
\text { difference }\end{array}$} & \multirow[b]{2}{*}{ Pooled SD } & \multirow[b]{2}{*}{$\begin{array}{l}\text { SD of paired } \\
\text { difference }\end{array}$} & \multicolumn{2}{|l|}{ Cohen's D } & \multicolumn{2}{|l|}{ SRM } & \multirow[b]{2}{*}{ Probability $(P \wedge)$} & \multirow[b]{2}{*}{$\begin{array}{l}95 \% \mathrm{Cl} \text { of } \\
\text { probability }\end{array}$} \\
\hline & & & & $\begin{array}{l}\text { Effect Size } \\
\text { (Cohen'd D) }\end{array}$ & $\begin{array}{l}95 \% \mathrm{Cl} \text { of } \\
\text { Cohen's D }\end{array}$ & SRM & $\begin{array}{l}95 \% \mathrm{Cl} \\
\text { of SRM }\end{array}$ & & \\
\hline $\begin{array}{l}\text { Assessment - Therapy } 1 \\
(N=134)\end{array}$ & 1.38 & 4.16 & 2.83 & 0.33 & $0.20-.46$ & 0.49 & $0.30-0.65$ & 0.69 & $0.65-0.72$ \\
\hline $\begin{array}{l}\text { Assessment - Therapy } 2 \\
(N=95)\end{array}$ & 3.04 & 4.55 & 4.41 & 0.67 & $0.48-0.86$ & 0.69 & $0.49-0.90$ & 0.75 & $0.71-0.78$ \\
\hline $\begin{array}{l}\text { Assessment - Therapy } 3 \\
(N=66)\end{array}$ & 3.75 & 4.07 & 4.21 & 0.92 & $0.69-1.15$ & 0.89 & $0.63-1.08$ & 0.81 & $0.77-0.84$ \\
\hline $\begin{array}{l}\text { Assessment - Therapy } 4 \\
(N=36)\end{array}$ & 4.37 & 4.24 & 4.32 & 1.03 & $0.60-1.41$ & 1.01 & $0.63-1.36$ & 0.84 & $0.80-0.88$ \\
\hline
\end{tabular}

Bootstrap confidence interval (1000 iterations) 
Table 3 Evaluation of responsiveness of SWEMWBS at individual level

\begin{tabular}{|c|c|c|c|c|c|c|c|}
\hline \multicolumn{5}{|c|}{ Large Improvement (Cohen's D > .5) } & \multicolumn{3}{|c|}{ SEM Improved > 2.77 SEM (2.87 points) } \\
\hline & $\mathrm{N}$ & $\mathrm{N}$ improved & $\%$ improved & $95 \% \mathrm{Cl}$ & $\mathrm{N}$ improved & $\%$ improved & $95 \% \mathrm{Cl}$ \\
\hline Assessment - Therapy 1 & 134 & 51 & 38.1 & $29.9-46.3$ & 37 & 27.6 & $20.1-35.8$ \\
\hline Assessment - Therapy 2 & 95 & 52 & 54.7 & $44.2-65.3$ & 46 & 48.4 & $38.9-58.9$ \\
\hline Assessment - Therapy 3 & 66 & 42 & 63.6 & $51.5-75.8$ & 35 & 53.0 & $40.9-65.2$ \\
\hline Assessment - Therapy 4 & 36 & 26 & 72.2 & $58.3-86.1$ & 24 & 66.7 & $50.0-80.6$ \\
\hline
\end{tabular}

each therapy session effect size and SRM produced more or less comparable values showing an increasing trend, providing corroboration of responsiveness via the two methods.

We considered change scores greater than 1 SEM [21] as well as 2.77 SEM [22] as cut off for statistically meaningful change at individual level as previous research has suggested both. The discrepancy between results might be due to differing methodologies, but could be explained by the fact that 2.77 SEM accounts for measurement error, combined variability across scores and chance at 95\% CI and therefore represents a higher threshold for meaningful change than 0.5 Cohen's D. It has also been suggested that thresholds of as low as 0.2 Cohen's D may be sufficient to demonstrate change [19]. Given this, the lower recommended threshold of 1 SEM (a change score of 1.03 points), or 2 SEM (change score of 2.06 points) which is close to the $95 \%$ confidence interval, rather than the change score of 2.87 suggested by $2.77 \mathrm{SEM}$ may be taken to denote statistically meaningful change.

\section{Strengths and limitations}

The study is based upon longitudinal data collected during practice of cognitive hypnotherapy with no control data. The changes could indicate regression to the mean or spontaneous improvement in mental health. As our objective was to determine whether SWEMWBS could detect changes in mental well-being for whatever reason, not whether this effect was due to administration of CHT, this is not of significant consequence to the analysis.

Crosby et. Al. have argued that an ideal assessment of responsiveness would involve integration of anchor based and distribution based techniques [14]. Responsiveness in this study was assessed using the distribution based methods only; and does not take into account the minimum important change as per the standards of programme participants, service users, carers or clinicians. Traditionally this would be done using a Global Rating of Change scale, although questions have been raised as to whether this method is valid for scales of mental wellbeing [27]. In the absence of an appropriate anchor, distribution methods are considered most appropriate $[21,25]$. Previous literature has suggested that an improvement of 0.5 units on each item on a Likert scale would equate to an improvement deemed important by individuals [28], which would equate to 3.5 SWEMWBS points which is higher than the threshold derived from even the most stringent tests in this study. Future studies using anchor based methods are need to refine these estimates and confirm the change score indicative of minimally important change from the perspective of study participants.

Studies comparing the responsiveness of SWEMWBS using the recommended two week as opposed to a one week response option are also needed to substantiate these findings.

\section{Implications for practice}

SWEMWBS is an attractive candidate for use as a clinical outcome measure due to its brevity and popularity with patients [6], allowing data to be easily collected in busy clinical settings. Despite the fact that SWEMWBS was originally developed to measure mental wellbeing at the population level, results indicate that it is responsive to change at both group and individual level in a clinical

Table 4 Improvement at lower SEM thresholds

\begin{tabular}{|c|c|c|c|c|c|c|c|}
\hline \multicolumn{5}{|c|}{$\begin{array}{l}\text { SEM } \\
\text { Improved at } 1 \text { SEM (1.03 points) }\end{array}$} & \multicolumn{3}{|c|}{$\begin{array}{l}\text { SEM } \\
\text { Improved at } 2 \text { SEM ( } 2.06 \text { points) }\end{array}$} \\
\hline & $\mathrm{N}$ & N improved & $\%$ improved & $95 \% \mathrm{Cl}$ & $\mathrm{N}$ improved & $\%$ improved & $95 \% \mathrm{Cl}$ \\
\hline Assessment - Therapy 1 & 134 & 69 & 51.5 & $43-59.8 \%$ & 51 & 38.1 & $30.2-46.5 \%$ \\
\hline Assessment - Therapy 2 & 95 & 66 & $69.5 \%$ & $59.6-77.8 \%$ & 56 & 58.9 & $48.9-68.3 \%$ \\
\hline Assessment - Therapy 3 & 66 & 49 & 74.2 & $62.6-83.2 \%$ & 42 & 63.6 & $51.5-74.1 \%$ \\
\hline Assessment - Therapy 4 & 36 & 26 & 72.2 & $56-84.1 \%$ & 28 & 77.8 & $61.9-88.2 \%$ \\
\hline
\end{tabular}


sample and in both group and individual analyses, responsiveness improved with prolonged therapy.

\section{Conclusion}

SWEMWBS is responsive to change at group level and individual level in a clinical sample of patients with depression and anxiety. Results using different standards suggest a difference of either 1 or 3 points as the threshold for statistically meaningful change at the individual level.

\section{Abbreviations}

$(\mathrm{P} \wedge)$ : Probability of change statistic; $\mathrm{CHT}$ : Cognitive Hypnotherapy Treatment; Cl: Confidence interval; ES: Effect Size; QCH: Quest Cognitive Hypnotherapy; SD: Standard Deviation; SEM: Standard Error of the Mean; SRM: Standardised Response Mean; SWEMWBS: Short Warwick-Edinburgh Mental Wellbeing Scale; WEMWBS: The Warwick-Edinburgh Mental Wellbeing Scale

\section{Acknowledgements}

We are grateful to Dr. Helen Parsons for reviewing early drafts of this manuscript and offering advice on approaches to statistical analyses. We are also grateful to the team at Quest Cognitive Hypnotherapy for providing the data.

\section{Funding}

No external funding was received.

\section{Availability of data and materials}

Restrictions apply to the availability of these data, which were used under data sharing agreement for the current study, and so are not publicly available. Data are however available from the authors upon reasonable request and with permission of Quest Cognitive Hypnotherapy.

\section{Authors' contributions}

SS-B, MC and NS designed the study. WA designed data collection. MC, NS and DW analysed the data. NS and SSB authored the manuscript. WA, NS, SSB, DW and MC reviewed, edited and approved the manuscript prior to submission for publication.

\section{Ethics approval and consent to participate}

Ethics approval has been granted from University of Warwick Biomedical and Scientific Research Ethics Sub-Committee, approval number REGO-2017-2066. Participating clients have all signed consent for their anonymised data to be used for research purposes.

\section{Consent for publication}

Not applicable.

\section{Competing interests}

Sarah-Stewart Brown is the developer of the Warwick-Edinburgh Mental Wellbeing Scale.

William Andrews is the developer of Pragmatic Tracker software used to capture data.

\section{Publisher's Note}

Springer Nature remains neutral with regard to jurisdictional claims in published maps and institutional affiliations.

\section{Author details}

${ }^{1}$ Public Health Registrar, Warwick Medical School University of Warwick, Coventry, UK. ${ }^{2}$ Consultant Community Physician, Ministry of Health, Colombo, Sri Lanka. ${ }^{3}$ Independent Researcher/ Consultant at Pragmatic Research Network, Rhosneigr, UK. ${ }^{4}$ Professor of Public Health, Warwick Medical School University of Warwick, Coventry, UK.
Received: 10 June 2018 Accepted: 4 December 2018

Published online: 22 December 2018

\section{References}

1. Faculty of Public Health and Mental Health Foundation. Better Mental Health for All: A Public Health Approach to Mental Health Improvement. 2016

2. Tennant R, Hiller L, Fishwick R, Platt S, Joseph S, Weich S, et al. The WarwickEdinburgh mental well-being scale (WEMWBS): development and UK validation. Health Qual Life Outcomes. 2007;5(1):63.

3. Deci EL, Ryan RM. Hedonia, eudaimonia, and well-being: an introduction. J Happiness Stud. 2008;9(1):1-11

4. Stewart-Brown S. In: Slade M, Jarden A, Oades L, editors. Population level: Wellbeing in the general population. Wellbeing: Recovery and Mental Health: CUP; 2017.

5. Stewart-Brown S, Tennant A, Tennant R, Platt S, Parkinson J, Weich S. Internal construct validity of the Warwick-Edinburgh mental well-being scale (WEMWBS): a Rasch analysis using data from the Scottish health education population survey. Health Qual Life Outcomes. 2009;7(1):15.

6. Crawford MJ, Robotham D, Thana L, Patterson S, Weaver T, Barber R, et al. Selecting outcome measures in mental health: the views of service users. J Ment Health (Abingdon, England). 2011:20(4):336-46.

7. Wilson IB, Cleary PD. Linking clinical variables with health-related quality of life: a conceptual model of patient outcomes. JAMA. 1995;273(1):59-65.

8. Higginson IJ, Carr AJ. Using quality of life measures in the clinical setting. BMJ. 2001:322(7297):1297-300.

9. Maheswaran H, Weich S, Powell J, Stewart-Brown S. Evaluating the responsiveness of the Warwick Edinburgh mental well-being scale (WEMWBS): group and individual level analysis. Health Qual Life Outcomes. 2012;10:156

10. Guyatt G, Walter S, Norman G. Measuring change over time: assessing the usefulness of evaluative instruments. J Chronic Dis. 1987;40(2):171-8.

11. Liang MH. Longitudinal construct validity: establishment of clinical meaning in patient evaluative instruments. Med Care. 2000;38(9 Suppl):li84-90.

12. Wyrwich KW, Bullinger M, Aaronson N, Hays RD, Patrick DL, Symonds T. Estimating clinically significant differences in quality of life outcomes. Qual Life Res. 2005;14(2):285-95.

13. Eisen SV, Ranganathan G, Seal P, Spiro A 3rd. Measuring clinically meaningful change following mental health treatment. J Behav Health Serv Res. 2007;34(3): 272-89.

14. Crosby RD, Kolotkin RL, Williams GR. Defining clinically meaningful change in health-related quality of life. J Clin Epidemiol. 2003;56(5):395-407.

15. Andrews WP, Parsons AA, Rawle $H$, Gibbs J. A pilot investigation of quest institute cognitive hypnotherapy services using improving access to psychological therapies as the benchmark. Ment Health Rev J. 2015;20(3): 199-210.

16. McHorney CA, Tarlov AR. Individual-patient monitoring in clinical practice: are available health status surveys adequate? Qual Life Res. 1995:4(4):293-307.

17. Middel B, van Sonderen E. Statistical significant change versus relevant or important change in (quasi) experimental design: some conceptual and methodological problems in estimating magnitude of intervention-related change in health services research. Int J Integr Care. 2002;2:e15.

18. Norman GR, Sloan JA, Wyrwich KW. Interpretation of changes in healthrelated quality of life: the remarkable universality of half a standard deviation. Med Care 2003:41(5):582-92

19. Lemieux J, Beaton DE, Hogg-Johnson S, Bordeleau LJ, Goodwin PJ. Three methods for minimally important difference: no relationship was found with the net proportion of patients improving. J Clin Epidemiol. 2007:60(5):448-55

20. Daly LE. Confidence limits made easy: interval estimation using a substitution method. Am J Epidemiol. 1998;147(8):783-90.

21. Wyrwich KW, Tierney WM, Wolinsky FD. Further evidence supporting an SEM-based criterion for identifying meaningful intra-individual changes in health-related quality of life. J Clin Epidemiol. 1999:52(9):861-73.

22. Jacobson NS, Truax P. Clinical significance: a statistical approach to defining meaningful change in psychotherapy research. J Consult Clin Psychol. 1991; 59(1):12.

23. Landis JR, Koch GG. The measurement of observer agreement for categorical data. Biometrics. 1977;33(1):159-74.

24. Fat LN, Scholes S, Boniface S, Mindell J, Stewart-Brown S. Evaluating and establishing national norms for mental wellbeing using the short Warwick- 
Edinburgh mental well-being scale (SWEMWBS): findings from the health survey for England. Qual Life Res. 2017;26:1129.

25. Zou GY. Quantifying responsiveness of quality of life measures without an external criterion. Qual Life Res. 2005;14(6):1545-52.

26. Norman G, Wyrwich KW, Patrick DL. The mathematical relationship among different forms of responsiveness coefficients. Qual Life Res. 2007;16(5):815-22.

27. Kamper SJ, Maher CG, Mackay G. Global rating of change scales: a review of strengths and weaknesses and considerations for design. J Man Manip Ther. 2009;17(3):163-70

28. Jaeschke R, Singer J, Guyatt GH. Measurement of health status: ascertaining the minimal clinically important difference. Control Clin Trials. 1989;10(4):407-15.

Ready to submit your research? Choose BMC and benefit from:

- fast, convenient online submission

- thorough peer review by experienced researchers in your field

- rapid publication on acceptance

- support for research data, including large and complex data types

- gold Open Access which fosters wider collaboration and increased citations

- maximum visibility for your research: over $100 \mathrm{M}$ website views per year

At $B M C$, research is always in progress.

Learn more biomedcentral.com/submissions 